\title{
Global Downscaling of Remotely-Sensed Soil Moisture using Neural Networks
}

Seyed Hamed Alemohammad ${ }^{1,2}$, Jana Kolassa ${ }^{3,4}$, Catherine Prigent ${ }^{1,2,5}$, Filipe Aires ${ }^{1,2,5}$, Pierre Gentine (1,2,6 $^{1}$

${ }^{1}$ Department of Earth and Environmental Engineering, Columbia University

$5 \quad{ }^{2}$ Columbia Water Center, Columbia University

${ }^{3}$ Universities Space Research Association, Columbia, MD

${ }^{4}$ Global Modelling and Assimilation Office, NASA Goddard Space Flight Center, Greenbelt, MD

${ }^{5}$ Observatoire de Paris

${ }^{6}$ Earth Institute, Columbia University

10 Correspondence to: Pierre Gentine (pg2328@ columbia.edu)

\section{Supplementary Materials}




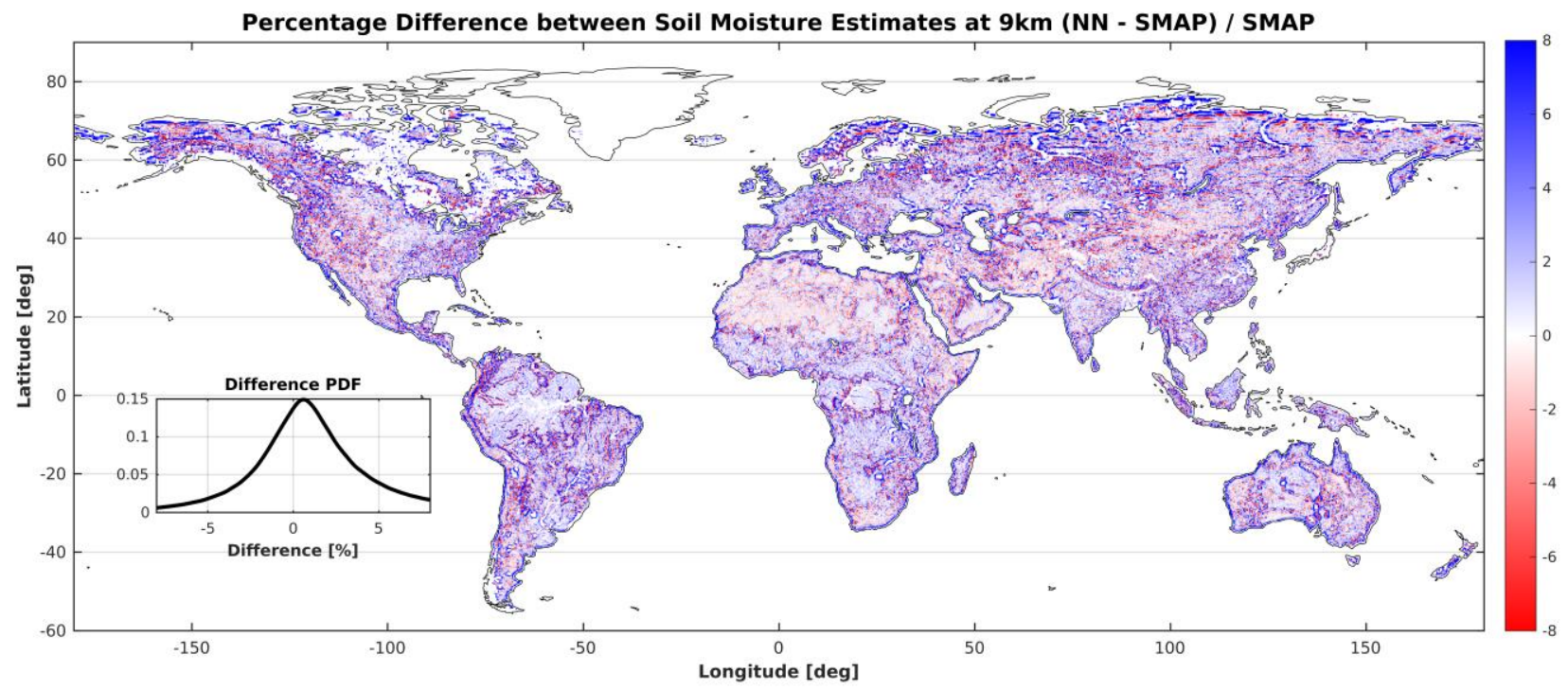

Figure S1- Percentage bias between the SMAP 9km soil mositure estimates and NN 9km estimates. White regions indicate no data. 


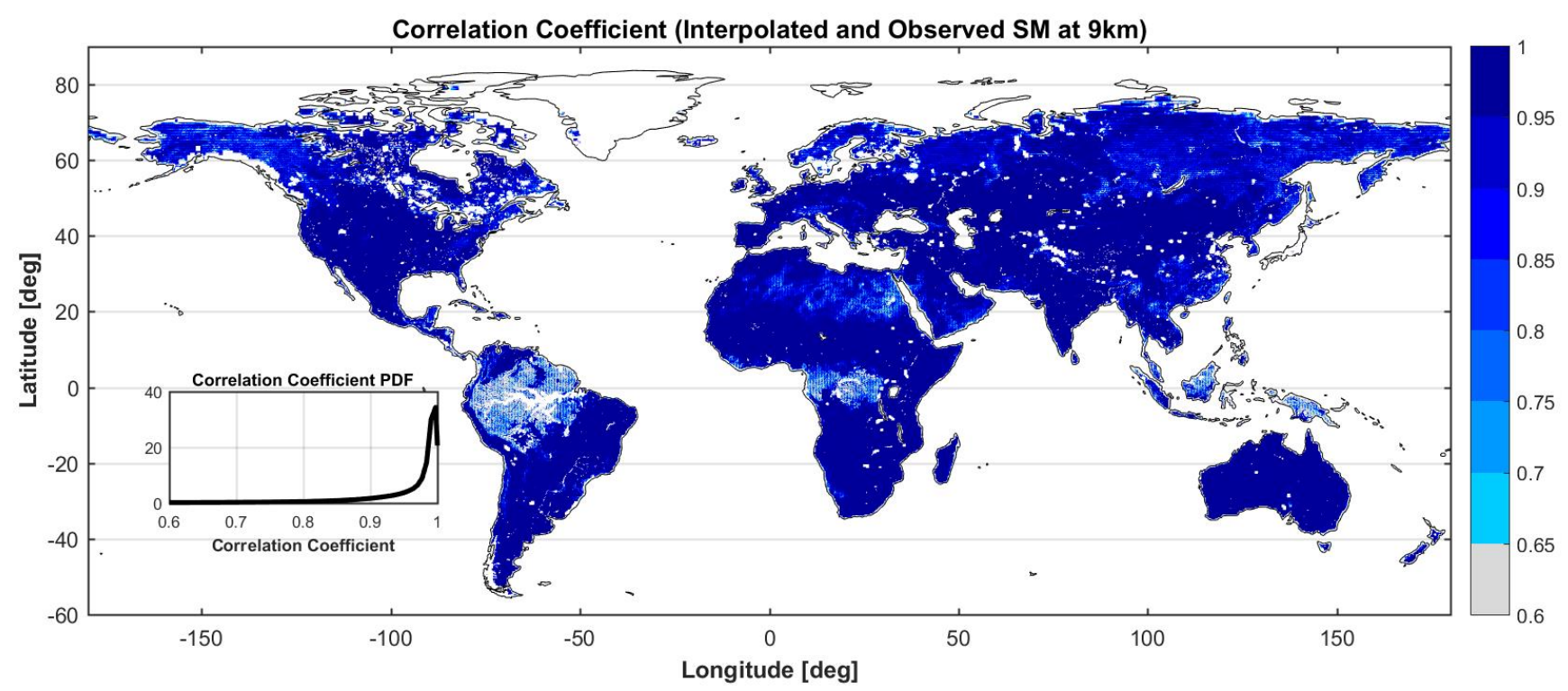

Figure S2- Correlation coefficient $\left(\mathbf{R}^{2}\right)$ between SMAP observed soil moisture at $9 \mathrm{~km}$ and Interpolated soil moisture at $9 \mathrm{~km}$. White regions indicate no data. 


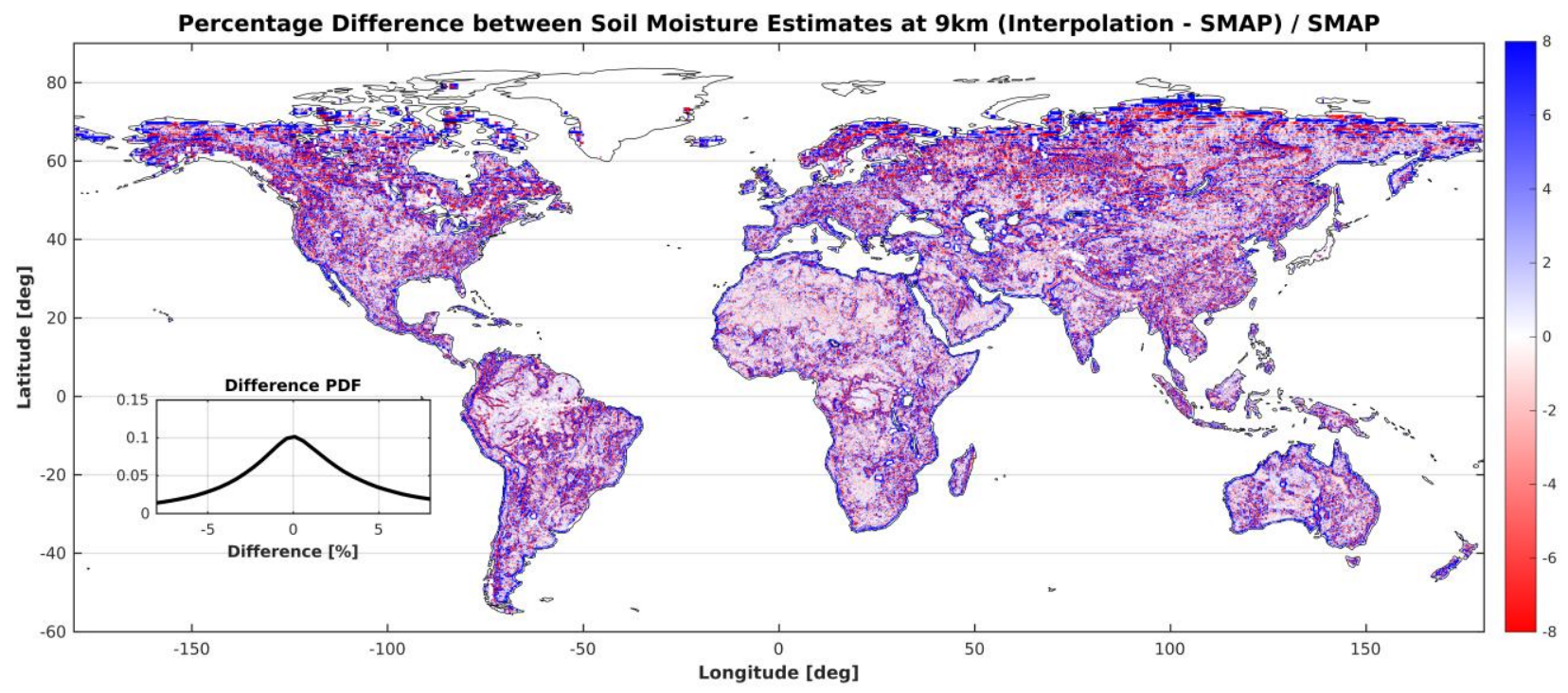

Figure S3- Percentage bias between the SMAP 9km soil mositure estimates and Interpolated 9km estimates. White regions indicate no data. 


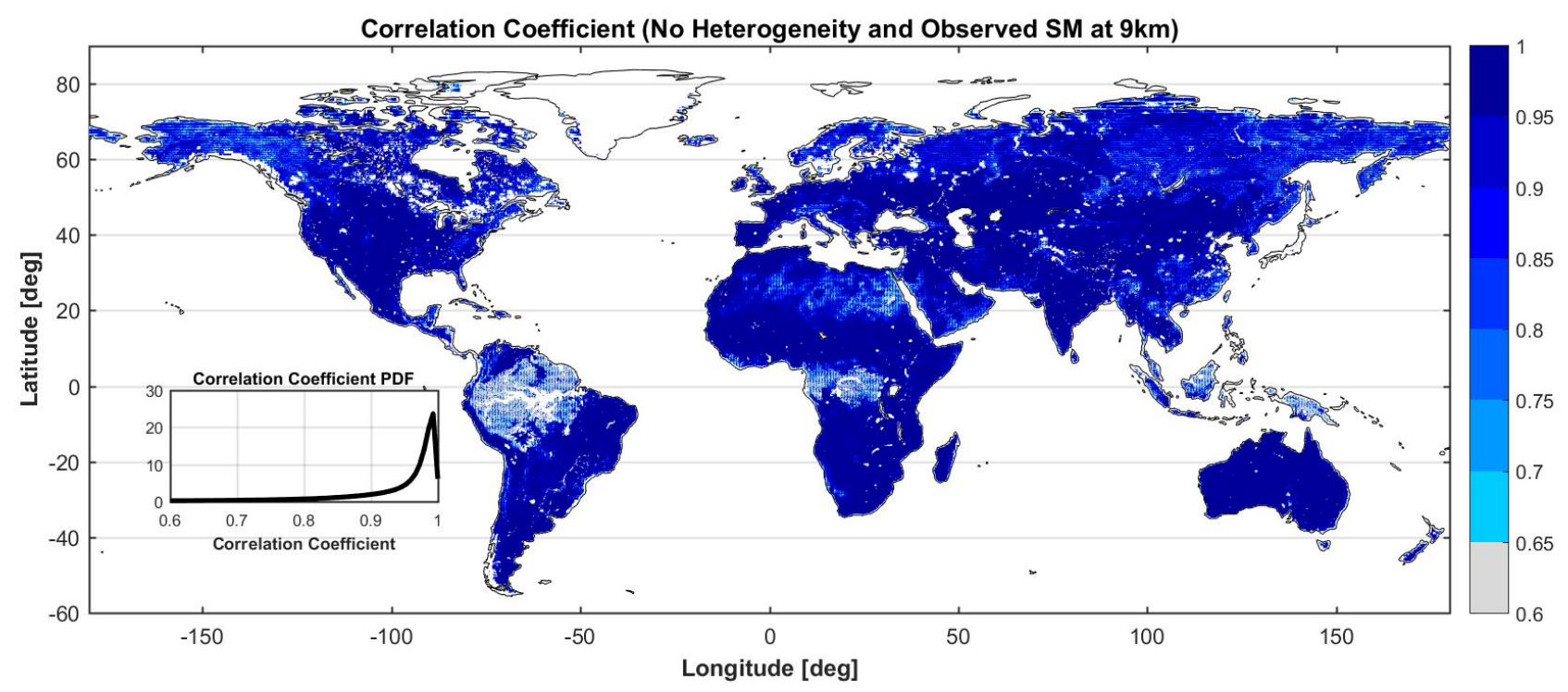

Figure S4- Correlation coefficient $\left(\mathbf{R}^{2}\right)$ between SMAP observed soil moisture at $9 \mathrm{~km}$ and No Heterogeneity soil moisture estimates at $9 \mathrm{~km}$. White regions indicate no data. 


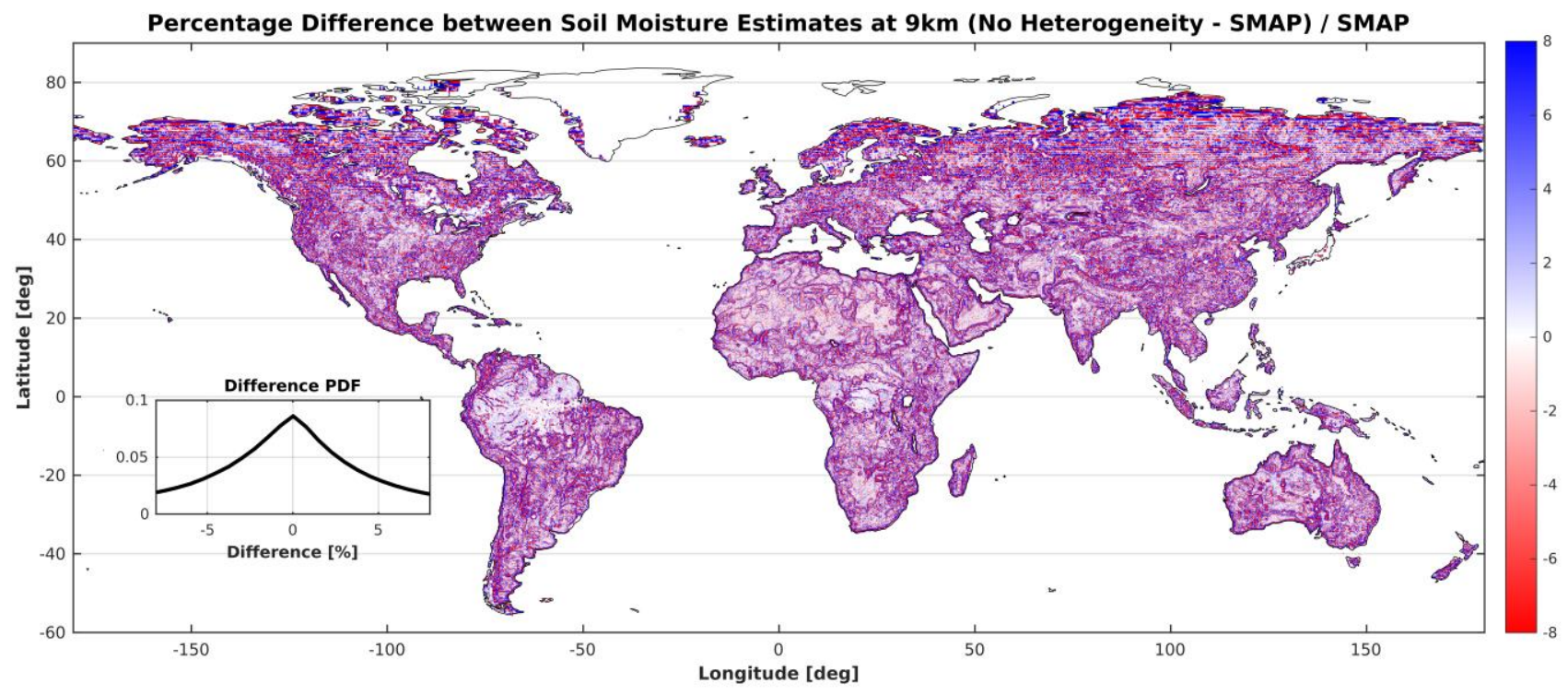

Figure S5- Percentage bias between the SMAP 9km soil mositure estimates and No Heterogeneity 9km estimates. White regions indicate no data. 


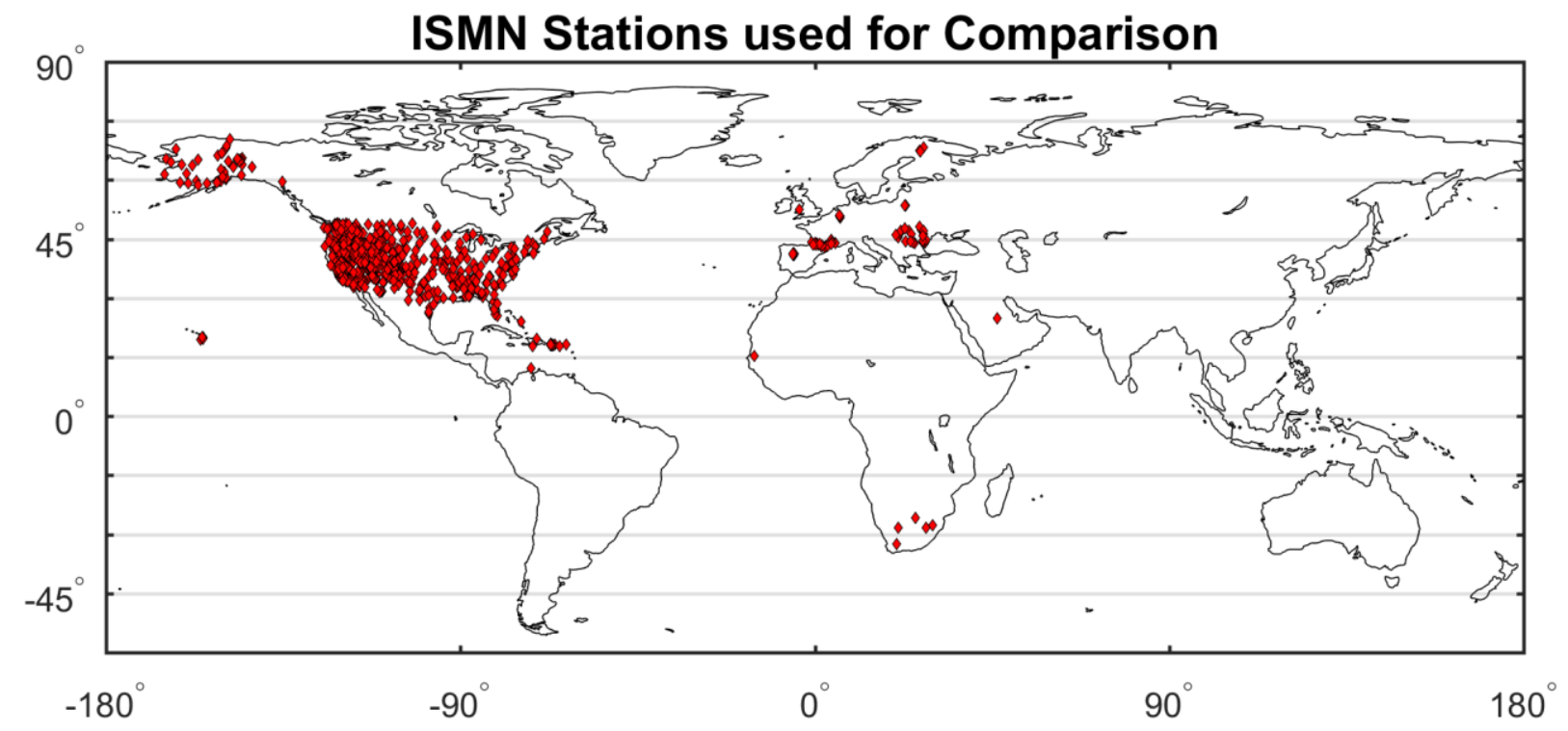

Figure S6- Location of each ISMN station used for comparison against downscaled soil moisture estimates 